\title{
Shear stress-induced improvement of red blood cell deformability
}

\author{
Ece Meram ${ }^{\mathrm{a}, *}$, Bahar D. Yilmaz ${ }^{\mathrm{a}, *}$, Ceren Bas ${ }^{\mathrm{a}, *}$, Nazlı Atac $^{\mathrm{a}}$, O. Yalcin ${ }^{\mathrm{a}}$, \\ Herbert J. Meiselman ${ }^{\mathrm{b}}$ and Oguz K. Baskurt ${ }^{\mathrm{a}, * *}$ \\ ${ }^{a}$ Koç University School of Medicine, Sariyer, Istanbul, Turkey \\ ${ }^{\mathrm{b}}$ Department of Physiology and Biophysics, Keck School of Medicine, \\ University of Southern California, Los Angeles, CA, USA
}

Received 14 April 2013

Accepted in revised form 28 May 2013

\begin{abstract}
Classically, it is known that red blood cell (RBC) deformability is determined by the geometric and material properties of these cells. Experimental evidence accumulated during the last decade has introduced the concept of active regulation of RBC deformability. This regulation is mainly related to altered associations between membrane skeletal proteins and integral proteins, with the latter serving to anchor the skeleton to the lipid matrix. It has been hypothesized that shear stress induces alterations of RBC deformability: the current study investigated the dynamics of the transient improvement in deformability induced by shear stress at physiologically-relevant levels. RBC were exposed to various levels of shear stress (SS) in a Couette type shearing system that is part of an ektacytometer, thus permitting the changes in RBC deformability during the application of SS to be monitored. Initial studies showed that there is an increase in deformability of the RBC subjected to SS in the range of 5-20 Pa, with kinetics characterized by time constants of a few seconds. Such improvement in deformability, expressed by an elongation index (EI), was faster with higher levels of SS and hence yielded shorter time constants: absolute values of EI increased by $3-8 \%$ of the starting level. Upon the removal of the shear stress, this response by RBC was reversible with a slower time course compared to the increase in EI during application of SS. Increased calcium concentration in the RBC suspending medium prevented the improvement of deformability. It is suggested that the improvement of RBC deformability by shear forces may have significant effects on blood flow dynamics, at least in tissues supplied by blood vessels with impaired vasomotor reserve, and may therefore serve as a compensating mechanism for the maintenance of adequate microcirculatory perfusion.
\end{abstract}

Keywords: Erythrocyte deformability, regulation, shear stress, ektacytometry, calcium

\section{Introduction}

Red blood cells (RBC) are exposed to various levels of shear stress (SS) during flow in the circulatory system $[20,36]$, with their passive response to these forces resulting in deformation and hence decreased in vivo flow resistance [13]. The ability of RBC to change their shape in response to given shear forces (i.e., deformability) determines their orientation with laminar flow streamlines and their ability to transverse the microcirculation, thereby affecting hemodynamic resistance in both large vessels (i.e., bulk flow) and the microcirculation [7]. In addition to this well-known mechanical behavior,

\footnotetext{
*These authors contributed equally to the study.

** Address for correspondence: Dr. Oguz K. Baskurt, Koç University School of Medicine, Sariyer, Istanbul, Turkey. Tel.: +90 212338 1160; Fax: +90 212338 1168; E-mail: obaskurt@ku.edu.tr.
} 
RBC have been shown to respond to effective shear forces by releasing adenosine triphosphate (ATP) via molecular mechanisms that have been described in detail $[33,40]$. RBC have also been shown to have enhanced generation and export of nitric oxide (NO) under physiological levels of SS [42,43]; both ATP and NO originating from RBC contribute to local vasomotor regulation [16]. Shear forces exceeding physiological levels may induce RBC damage ranging from alterations in cellular properties (e.g., reduced deformability) to hemolysis [22]. RBC can withstand SS up to $300 \mathrm{~Pa}$ for short periods $(<1 \mathrm{~s})$ without hemolysis [24], yet SS in the range of 100-300 Pa can induce mechanical impairment which is detectable as reduced deformability and/or stability $[9,29]$.

RBC deformability is classically known to be determined by the geometric and material properties of these cells $[7,13,28]$. Interestingly, preliminary observations of the "active" responses of RBC to SS have suggested that these responses may play important roles in the regulation of RBC properties, including deformability. Detailed proteomic studies show that RBC possess the elements of a wide variety of intracellular signaling mechanisms and related protein kinases and phosphatases [19]. Furthermore, several modulators of intracellular signaling have been demonstrated to influence RBC deformability $[11,27,30]$. Additionally, protein interactions have been suggested to be controlled by post-translational modifications (e.g., phosphorylation status) via these signaling pathways, providing the background for alterations in RBC mechanical properties [17,38]. These observations suggest the possibility that SS may trigger changes in signaling mechanisms and their targets, leading to various functional alterations including the modulation of RBC mechanical properties. Such effects of SS on the functions of other types of cells (e.g., endothelial cells, platelets) have been demonstrated [14,37]. However, experimental data for possible shear stress-induced activation of signaling mechanisms, as well as for alterations of mechanical properties, are not available for human RBC.

The present study was designed to test the hypothesis that physiological levels of shear stress acting on RBC lead to alterations of their mechanical properties. In particular, the focus was on investigating and defining the basic aspects of relations between applied shear stress and RBC deformability.

\section{Materials and methods}

\subsection{Blood samples}

Venous blood samples were obtained from healthy human volunteers, aged between 19 to 57 years, using vacuum tubes containing sodium heparin $(15 \mathrm{IU} / \mathrm{ml})$. A tourniquet was applied to the upper arm during the sampling from the antecubital vein and the sampling was completed within $90 \mathrm{~s}$ following application of the tourniquet [2]. All experiments were conducted within four hours following the sampling.

\subsection{Preparation of red blood cell suspensions}

RBC suspensions were prepared by diluting whole blood, at a 1:200 ratio, in viscous solutions of $12 \%$ high molecular mass dextran (500 kDa, Sigma Chemical Co., St. Louis, MO) dissolved in isotonic phosphate buffer (PBS, $\mathrm{pH}=7.4,290 \mathrm{mOsm} / \mathrm{kg}$ ). The viscosity of the $12 \%$ dextran solution was $23.6 \mathrm{mPa} \cdot \mathrm{s}$ at $37^{\circ} \mathrm{C}$. A calcium chelator (ethylenediaminetetraaceticacid, EDTA) at $2 \mathrm{mg} / \mathrm{ml}(6.8 \mathrm{mM})$ or calcium chloride at 1-5 mM were added to the dextran solution for some experiments. The RBC in the dextran solutions were exposed to shear stress using the ektacytometry system described in Section 2.3. 


\subsection{Application of shear stress to RBC suspensions}

RBC suspensions were exposed to constant shear stresses between 5 to $20 \mathrm{~Pa}$ using a laser diffraction ektacytometer system (LORCA MaxSis, Mechatronics, Hoorn, The Netherlands). The system contains a co-axial, Couette cylindrical shearing system; both the inner and outer cylinders are made of transparent material with a gap of $\sim 350 \mu \mathrm{m}$ between them. The outer cylinder rotates at a calculated rotational speed to generate the desired SS in the sample introduced to this gap; the SS in the gap was obtained using the rotational speed of the outer cylinder and the viscosity of the suspending medium $(23.6 \mathrm{mPa} \cdot \mathrm{s})$. A laser beam ( $670 \mathrm{~nm}$ wavelength) is projected perpendicular to the axis of rotation and the diffraction pattern generated by the RBC is projected onto a screen located after the beam passes through the gap. The pattern is captured by a CCD camera and analyzed by an attached computer which also controls the rotational speed of the outer cylinder. RBC elongation indices (EI), calculated by using the long and short axes of the elliptical diffraction patterns ( $a$ and $b$, respectively) as EI $=(a-b) /(a+b)$, were continuously monitored during the application of SS. The experiments were conducted at $37^{\circ} \mathrm{C}$.

\subsection{Assessment of red blood cell deformability}

Two experimental protocols were used to assess RBC deformability: (1) RBC elongation indices were determined at 10 different SS between 0.3 to $50 \mathrm{~Pa}$ using the ektacytometer described in Section 2.3 and its automated measurement routine. EI-SS measurements were made both before and after the continuous application of selected levels of SS to the RBC suspensions. The EI-SS determination after the SS application was initiated following a full-stoppage of the outer cylinder of the ektacytometer, with a delay of $5 \mathrm{~s}$ or less. Data are expressed as EI-SS curves. These EI-SS data were characterized by the maximum EI at infinite $\mathrm{SS}\left(\mathrm{EI}_{\max }\right)$ and the SS needed to achieve one-half of this maximum $\left(\mathrm{SS}_{1 / 2}\right)$ using the Lineweaver-Burk approach and non-linear curve fitting [3]; the $\mathrm{SS}_{1 / 2} / \mathrm{EI}_{\max }$ ratio was calculated as a normalized measure of $\mathrm{SS}_{1 / 2}$ [6]; (2) RBC were subjected to constant shear stress of 5, 10 or $20 \mathrm{~Pa}$ and EI-time data were obtained during this period. For some experiments, EI-time data were recorded at $5 \mathrm{~Pa} \mathrm{SS}$ for 10, 20 or $30 \mathrm{~s}$, then the recording was repeated at the same $5 \mathrm{~Pa}$ shear stress following a waiting time of 10 or $60 \mathrm{~s}$.

\subsection{Data presentation and statistics}

Data are presented as mean \pm standard error (SE). Two-way ANOVA followed by Bonferoni posttest were used for comparisons between before and after measurements. The $\mathrm{SS}_{1 / 2} / \mathrm{EI}_{\max }$ parameter and ratios of data (Table 1) were compared using a paired t test for comparisons between two groups and one-way ANOVA for multi-group comparisons. Non-linear curve fitting for analysis of the time course of EI changes and the Lineweaver-Burk approach to calculate $\mathrm{SS}_{1 / 2}$ and $\mathrm{EI}_{\max }$ were performed by GraphPad 4.0 software package (GraphPad Software, La Jolla, CA).

\section{Results}

\subsection{Elongation indexes before and after shear stress application}

Figure 1 presents EI-SS curves obtained before and after the application of 5 Pa SS to RBC suspensions. Two-way ANOVA indicated a significant difference between these two curves; further analysis 


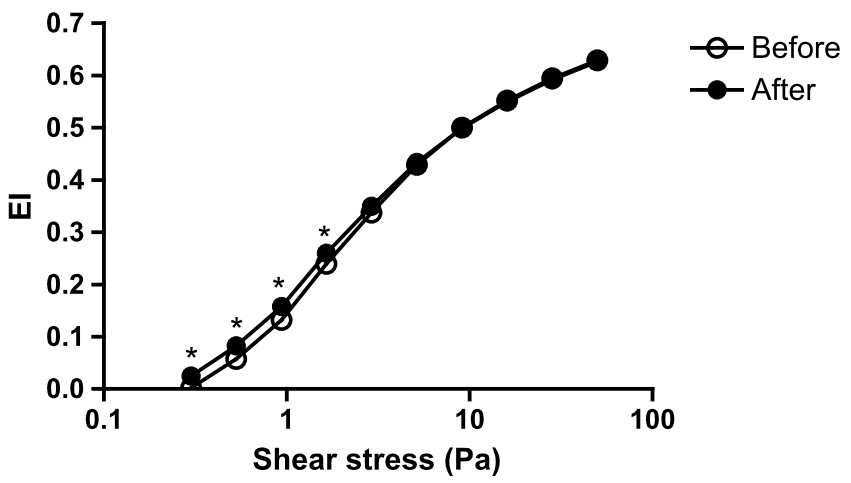

Fig. 1. Shear stress-elongation index (EI) curves obtained before and after the application of $5 \mathrm{~Pa}$ shear stress to red blood cell suspensions for $300 \mathrm{~s}$. The after curves were obtained after a $5 \mathrm{~s}$ delay following the end of the 5 Pa SS application period. The data are mean $\pm \mathrm{SE}$ for 10 experiments; error bars remain within the symbols for all data points. The two curves differ significantly (two-way ANOVA) with differences between before and after at each SS tested by the Bonferroni post-test. Difference from before ${ }^{*}: p<0.05$.

(a) $5 \mathrm{~Pa}$ SS applied for 300 seconds



(b) $5 \mathrm{~Pa}$ SS applied for 30 seconds

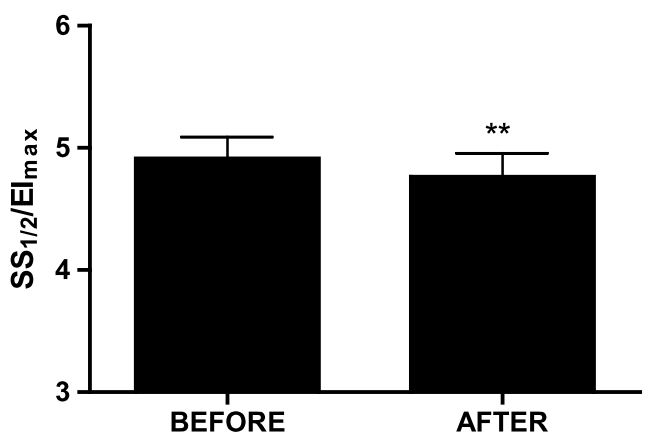

Fig. 2. $\mathrm{SS}_{1 / 2} / \mathrm{EI}_{\max }$ parameters calculated for EI-SS data obtained before and after the application of $5 \mathrm{~Pa} \mathrm{SS}$ for (a) $300 \mathrm{~s}$ and (b) $30 \mathrm{~s}$. After data refer to the measurements with $5 \mathrm{~s}$ delay following cessation of the SS application. Difference from before ${ }^{* *} p<0.01 ;{ }^{* * *} p<0.001$.

using the Bonferroni post-test at each SS indicated significant increases of EI between 0.30 to $5.15 \mathrm{~Pa}$ SS.

Figure 2(a) presents $\mathrm{SS}_{1 / 2} / \mathrm{EI}_{\max }$ values calculated using the EI-SS data shown in Fig. 1. Following the application of $5 \mathrm{~Pa}$ SS for $300 \mathrm{~s}$ there was a significant $12 \%$ improvement in RBC deformability as reflected in a significant decrease of $\mathrm{SS}_{1 / 2} / \mathrm{EI}_{\max }$ from that before SS application (4.90 $\left.\pm 0.16 \mathrm{~Pa}\right)$ to that after SS $(4.29 \pm 0.20 \mathrm{~Pa}) ;(p<0.001)$. In a second series of experiments in which the application of $5 \mathrm{~Pa}$ was reduced to $30 \mathrm{~s}$, the magnitude of the improvement was smaller but still significant (Fig. 2(b)): $\mathrm{SS}_{1 / 2} / \mathrm{EI}_{\max }$ was $4.91 \pm 0.17 \mathrm{~Pa}$ before SS application and $4.76 \pm 0.19 \mathrm{~Pa}$ after 30 seconds SS - a $3 \%$ improvement $(p<0.01)$.

\subsection{Time course of the change in elongation indexes under shear stress}

Figure 3(a) presents a typical time course of EI during the application of $5 \mathrm{~Pa}$ SS for $300 \mathrm{~s}$, and Fig. 3(b) shows the mean of 10 separate experiments focusing on the first $30 \mathrm{~s}$ following the start of 
(a)

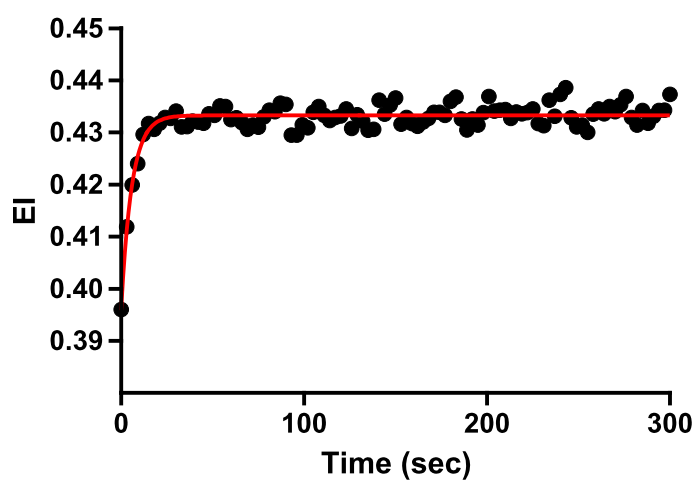

(b)

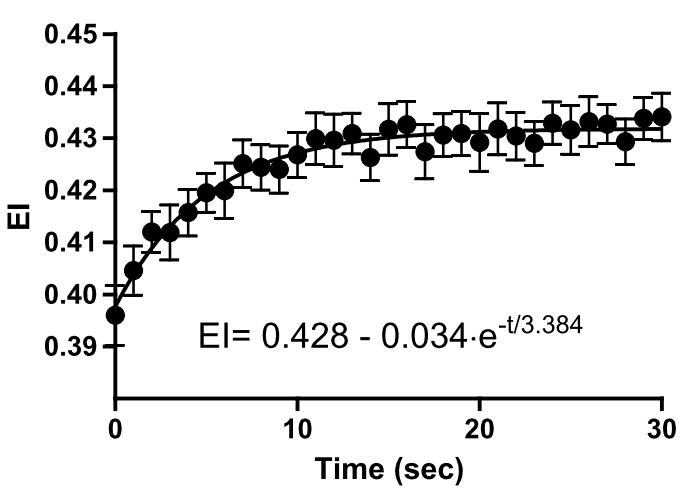

Fig. 3. Time course of elongation index (EI) changes in response to the application of 5 Pascal SS for $300 \mathrm{~s}$. Panel (a) presents a typical example, while panel (b) presents the mean \pm SE of 10 separate experiments during the first $30 \mathrm{~s}$. The curve shown in panel (b) was fitted using a least-squares approximation method (GraphPad Software). (Colors are visible in the online version of the article; http://dx.doi.org/10.3233/BIR-130637.)

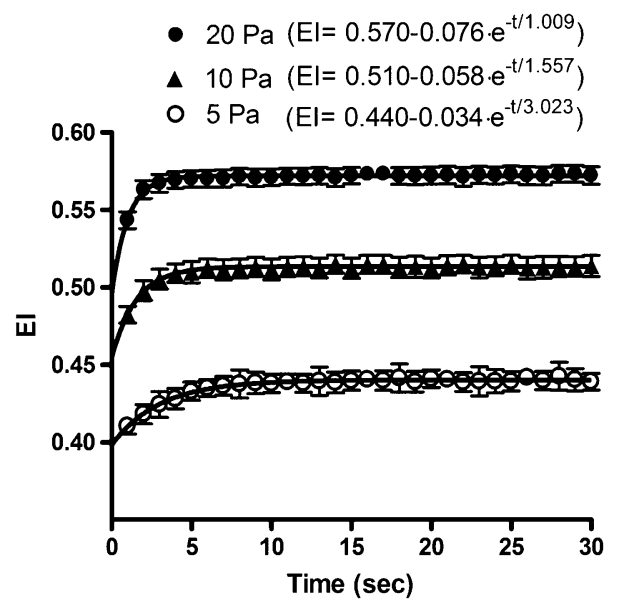

Fig. 4. EI-time results for RBC subjected to 5, 10 and 20 Pa shear stress. Data points are mean \pm SE of 10 experiments using different blood samples. The equations were obtained using a least squares approximation method. Note that the time constant varies inversely with the magnitude of stress.

SS application. The data can be fitted to an exponential equation and yield the following characteristic information: (1) The increase of EI follows an exponential rise with a time constant of $3.384 \mathrm{~s}$; (2) EI reaches a plateau level of 0.428 within the first $10-15 \mathrm{~s}$; (3) The total increase from the initial value to this plateau is 0.034 (EI) and thus a $8.6 \%$ rise above the initial EI.

Figure 4 presents EI-time results for RBC during $30 \mathrm{~s}$ of shear at 5, 10 or $20 \mathrm{~Pa}$ in a separate set of experiments. Salient findings were: (1) at $5 \mathrm{~Pa}$, EI increased $\sim 7.0 \%$ to a plateau of $0.440 \pm 0.007$ with a time constant of $3.023 \pm 0.046 \mathrm{~s}$; (2) at $10 \mathrm{~Pa}$, EI increased $\sim 6.4 \%$ to a plateau of $0.510 \pm 0.006$ with a time constant of $1.557 \pm 0.028 \mathrm{~s}$; (3) at $20 \mathrm{~Pa}$, EI increased $\sim 5.3 \%$ to a plateau of $0.570 \pm 0.006$ with a time constant of $1.009 \pm 0.024 \mathrm{~s}$. Thus, the time constant was inversely related to the level of applied shear stress. 


\subsection{Reversibility of elongation index improvement following shear stress application}

The reversibility of the increase in EI after abruptly stopping the application of SS was evaluated by repeating EI measurements after waiting 10 or $60 \mathrm{~s}$ following a 10, 20 or $30 \mathrm{~s}$ application of $5 \mathrm{~Pa} \mathrm{SS}$ (Fig. 5). These results indicate that the improvement of EI is partly reversible at $10 \mathrm{~s}$ regardless of the length of time the $5 \mathrm{~Pa}$ SS was applied and that the reversal of EI improvement was greater when the wait time was $60 \mathrm{~s}$. Figure 5(a) clearly illustrates these observations: (1) Applying SS for $10 \mathrm{~s}$ increased EI from $0.393 \pm 0.003$ to $0.417 \pm 0.004$ (6.1\% increase), following which EI decreased at $10 \mathrm{~s}$ to $0.407 \pm 0.004$ and hence a $41.7 \%$ reversal (Fig. 5(a)); (2) Applying SS for $10 \mathrm{~s}$ but extending the waiting time to $60 \mathrm{~s}$ decreased EI to $0.403 \pm 0.003$ corresponding to a $64 \%$ reversal. Results for 20 and $30 \mathrm{~s}$ application of $5 \mathrm{~Pa}$ and 10 or $60 \mathrm{~s}$ wait time are shown in Fig. 5(b) and (c) and in Table 1. It is interesting to note that the reversal ratio was lower if the $5 \mathrm{~Pa}$ SS exposure time was $30 \mathrm{~s}$, especially with a $60 \mathrm{~s}$ waiting time.

(a)

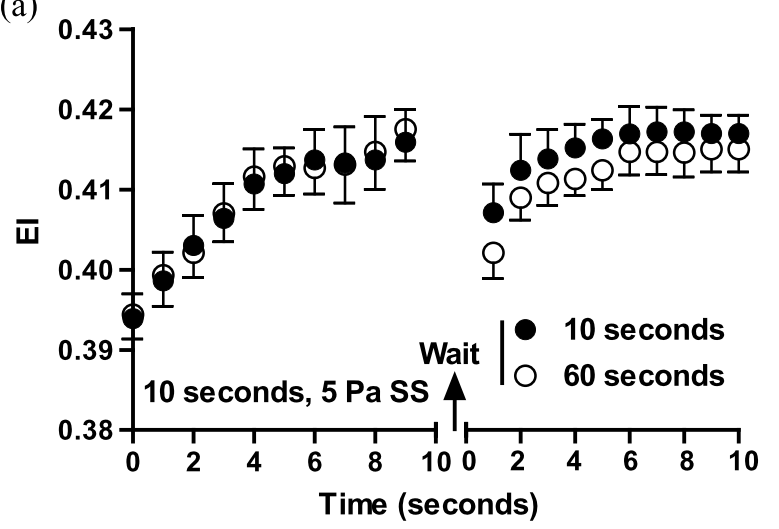

(b)

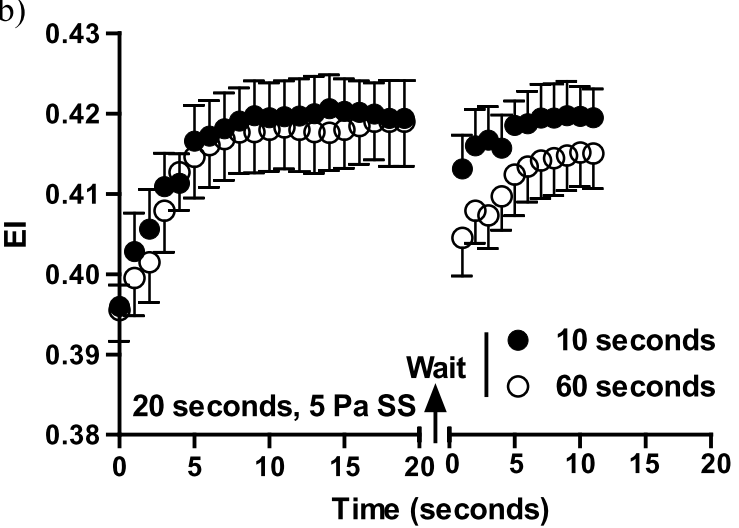

(c)

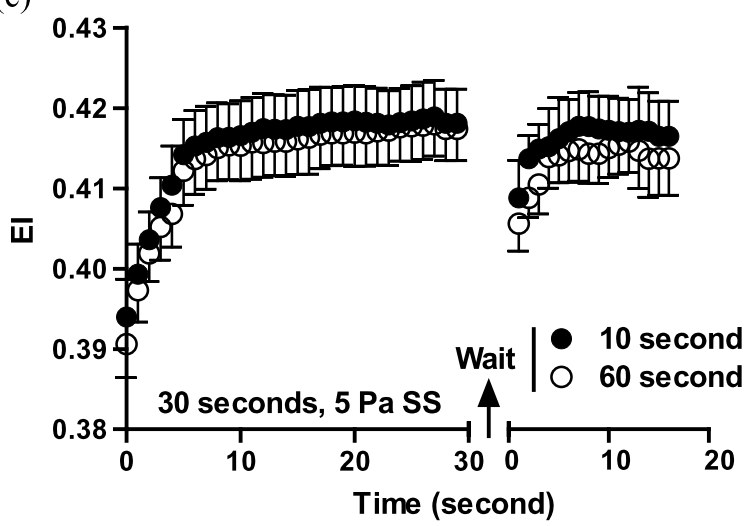

Fig. 5. Reversibility of the improvement in elongation indexes (EI). A shear stress of 5 Pa was applied to RBC suspensions for 10,20 or $30 \mathrm{~s}$ (panels (a), (b) and (c), respectively), then EI measurements repeated at 5 Pa following 10 or $60 \mathrm{~s}$ waiting time. The 10 and $60 \mathrm{~s}$ results were obtained in separate studies. The data are statistically compared in Table 1 . 
Table 1

Red blood cell elongation indexes (EI) during the first application of 5 Pa shear stress for periods of 10, 20 and $30 \mathrm{~s}$ and during the repeated application of the same shear stress after 10 or $60 \mathrm{~s}$ following cessation of the first shearing

\begin{tabular}{|c|c|c|c|c|}
\hline Shearing time at $5 \mathrm{~Pa}$ & & $10 \mathrm{~s}$ & $20 \mathrm{~s}$ & $30 \mathrm{~s}$ \\
\hline \multirow[t]{3}{*}{ First shearing } & $\mathrm{EI}_{\text {initial }}$ & $0.394 \pm 0.002$ & $0.396 \pm 0.002$ & $0.392 \pm 0.003$ \\
\hline & $\mathrm{EI}_{\text {final }}$ & $0.417 \pm 0.003$ & $0.419 \pm 0.003$ & $0.417 \pm 0.003$ \\
\hline & Improvement $(\%)$ & $5.7 \pm 0.4$ & $5.9 \pm 0.5$ & $6.5 \pm 0.4^{*}$ \\
\hline \multirow{3}{*}{$\begin{array}{l}\text { Second shearing } \\
\quad \text { (after } 10 \mathrm{~s})\end{array}$} & $\mathrm{EI}_{\text {initial }}$ & $0.407 \pm 0.003$ & $0.413 \pm 0.004$ & $0.409 \pm 0.005$ \\
\hline & $\mathrm{EI}_{\text {final }}$ & $0.417 \pm 0.002$ & $0.420 \pm 0.004$ & $0.417 \pm 0.004$ \\
\hline & Improvement (\%) & $2.4 \pm 0.4$ & $1.6 \pm 0.2$ & $2.1 \pm 0.4$ \\
\hline \multirow{3}{*}{$\begin{array}{l}\text { Second shearing } \\
\quad(\text { after } 60 \mathrm{~s})\end{array}$} & $\mathrm{EI}_{\text {initial }}$ & $0.402 \pm 0.003$ & $0.404 \pm 0.004$ & $0.405 \pm 0.003$ \\
\hline & $\mathrm{EI}_{\text {final }}$ & $0.415 \pm 0.003$ & $0.415 \pm 0.004$ & $0.415 \pm 0.003$ \\
\hline & Improvement (\%) & $3.2 \pm 0.2^{* *}$ & $2.6 \pm 0.3^{* *}$ & $2.3 \pm 0.4$ \\
\hline Reversal in $10 \mathrm{~s}(\%)$ & & $41.7 \pm 11.2$ & $26.8 \pm 5.07$ & $40.0 \pm 9.3$ \\
\hline Reversal in $60 \mathrm{~s}(\%)$ & & $67.5 \pm 4.2^{* *}$ & $58.9 \pm 8.1^{* *}$ & $45.3 \pm 6.4$ \\
\hline
\end{tabular}

Data derived from Fig. 5(a), (b) and (c).

$\mathrm{EI}_{\text {initial }}$ is the initial value of EI immediately following the SS application; $\mathrm{EI}_{\text {final }}$ is the plateau value after the curve fitting (see Fig. 3); Improvement $(\%)$ calculated as $\left[\left(\mathrm{EI}_{\text {final }}-\mathrm{EI}_{\text {initial }}\right) / \mathrm{EI}_{\text {initial }}\right] \times 100$; Reversal $(\%)$ calculated as $\left[\left[\left(\mathrm{EI}_{\text {final }}\right.\right.\right.$, first shearing $)-$ $\left(\mathrm{EI}_{\text {initial }}\right.$, second shearing $\left.)\right] /\left(\mathrm{EI}_{\mathrm{final}}-\mathrm{EI}_{\text {initial }}\right.$, first shearing $\left.)\right] \times 100$. Data are mean \pm standard error of 10 experiments.

*Difference from "10 s shearing time", $p<0.05$; ${ }^{* *}$ Difference from " 10 seconds waiting time", $p<0.05$.

\subsection{Influence of calcium content of the suspending medium}

Figure 6(a) presents EI-time results at $5 \mathrm{~Pa}$ SS for RBC suspended in the viscous dextran medium containing EDTA $(6.8 \mathrm{mM})$ or $\mathrm{CaCl}_{2}(1-5 \mathrm{mM})$. Figure 6(b) shows the percent change in EI, calculated as $\left[\left(\mathrm{EI}_{\text {final }}-\mathrm{EI}_{\text {initial }}\right) / \mathrm{EI}_{\text {initial }}\right] \times 100$, where $\mathrm{EI}_{\text {initial }}$ is the $\mathrm{EI}$ measured at the beginning of the application of SS and $\mathrm{EI}_{\text {final }}$ is the plateau value. EI increased $5.9 \pm 0.6 \%$ during the $30 \mathrm{~s}$ shearing period for the control experiments in which the RBC suspension did not contain EDTA or additional calcium. Removal of ionized calcium from the suspending medium by EDTA enhanced the increase of EI to $8.5 \pm 0.5 \%$ ( $p<0.01$ versus control). Addition of $2.5 \mathrm{mM}$ calcium to the suspending medium resulted in a lower change $(2.0 \pm 0.8 \%)$ that was significantly different from control $(p<0.01)$. For cells in the medium containing $5 \mathrm{mM}$ calcium, EI decreased with time (Fig. 6).

\section{Discussion}

The response of the RBC to external forces in terms of shape change is a well-documented phenomenon; this ability is usually quantified by an elongation index, EI [21]. EI under a constant SS or alterations of EI over a given range of SS (i.e., deformability) reflect RBC properties affecting their mechanical response to fluid shear stress. The novel finding of the present study is the progressive improvement of deformability during the application of physiological levels of SS [20] (i.e., increased EI over time under a constant SS). This response of RBC is fast enough to be physiologically meaningful, and at 5-20 Pa is characterized by time constants of only $\sim 1-3 \mathrm{~s}$ (Fig. 4). Further, it has been observed that this response is reversible upon the removal of SS, again within time periods on the order of seconds, thus indicating the dynamic nature of the response that is consistent with microcirculatory passage and circulation times [26].

The time course of the change in EI at a given SS in an ektacytometer is affected by three factors: (1) the time constant for the cup system to reach to steady rotational speed; (2) the time constant for 
(a)

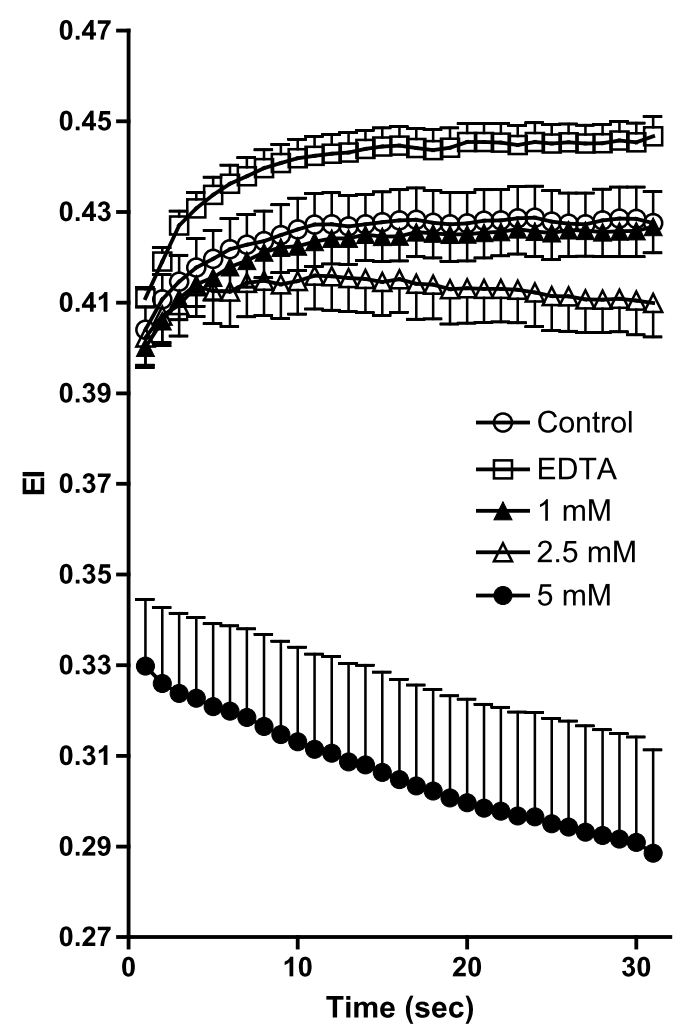

(b)

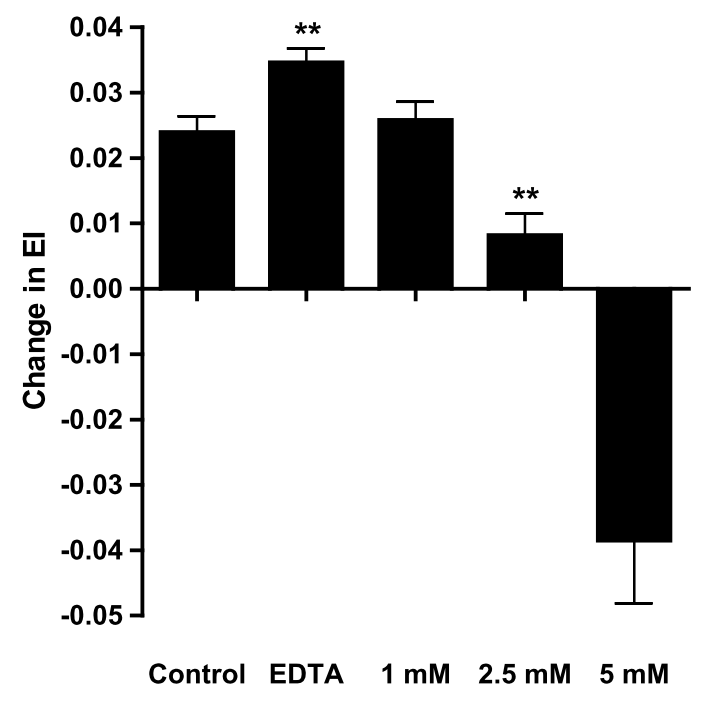

Fig. 6. Effect of calcium content of the suspending medium on the elongation index (EI) alterations during shear stress (SS). RBC suspensions were exposed to $5 \mathrm{~Pa} \mathrm{SS}$ in the presence of $6.8 \mathrm{mM}$ EDTA or $1-5 \mathrm{mM} \mathrm{CaCl}_{2}$. (a) EI-SS curves. The data points on the curves are mean \pm SE of 10 experiments. (b) Percent changes in EI at $5 \mathrm{~Pa}$ SS over the 30 s period. (Please see Section 3.4 for the explanation of calculation). Difference from control, ${ }^{* *} p<0.01$.

establishing the steady-state velocity profile in the gap between the bob and cup; (3) the time constant for the RBC to deform in response to fluid SS. However, these factors seem unlikely to have affected our experimental findings: (1) the cup is driven by a servo-controlled, low inertia, high-torque pancake motor whose dynamic behavior is sufficiently fast; (2) the high suspending phase viscosity (i.e., $23.6 \mathrm{mPa} \cdot \mathrm{s}$ ) and hence high kinematic viscosity predict that the velocity profile becomes fully developed in less than $100 \mathrm{~ms}$ [39]; (3) the characteristic RBC response time is on the order of 100-150 ms [8]. It thus seems reasonable to assume that our ektacytometer findings indicate RBC equilibrium deformation at a given SS for any time point beyond $\sim 0.2 \mathrm{~s}$. Further, the very significant differences in the time constants measured in the suspensions with different calcium contents under the same SS (Fig. 6) also support the notion that EI-time relations reflect cellular behavior, as calcium levels affect RBC mechanical properties $[5,18,32]$ yet do not alter the fluid mechanics of the system.

The mechanisms involved in the observed improvement in EI have not been fully detailed in the present study. Earlier reports of EI-time relations, mainly of RBC membrane ghosts, at higher SS $(<50 \mathrm{~Pa})$ for prolonged periods indicated an initial increase which was followed by a significant reduction in EI $[12,29]$. These alterations in membrane properties were attributed to mechanical breakdown of the membrane skeletal network due to prolonged near-maximal linear extension [12]. However, such a 
mechanism seems unlikely for the observed improvement of deformability in the present study since the SS regime used (i.e., 5-20 Pa for shorter periods) is not expected to induce linear extension of the cells close to the maximum. Furthermore, the reversibility of the response also does not support a breakdown mechanism.

Interestingly, reversal of EI improvement was not complete even after a $60 \mathrm{~s}$ delay following the initial shearing period, although the reversal was significantly greater following $60 \mathrm{~s}$ if the initial shearing period was below $20 \mathrm{~s}$. This behavior may indicate response mechanisms related to biochemical-structural alterations, with "products" accumulating during the SS application. The reduced magnitude of the improvement following a delay time of $60 \mathrm{~s}$ also supports this reasoning: some capacity of the mechanisms contributing to the improvement of RBC deformability might be utilized during the first shearing and this capacity could not be restored during the relatively short delay time. This pattern suggests that signaling mechanisms, together with their targets (e.g., protein phosphorylation/dephosphorylation systems) that are known to play a role in the regulation of RBC mechanical properties $[11,27,30]$, may also be involved in the RBC changes noted herein. However, it still remains to be experimentally explored whether the timing of these signaling cascades can explain the relatively fast response of RBC deformability.

Contributions of intracellular signaling mechanisms to the observed response were not evaluated in this study. However, observations related to the influence of calcium in the suspending medium during SS application support the role of such signaling cascades in RBC deformability. It is notable that the presence of calcium, even at its physiological concentration $(2.5 \mathrm{mM})$, blunted the deformability improvement response under SS; higher concentrations of calcium resulted in reversal of the effect, with EI decreasing with time during SS (Fig. 6). It has been previously demonstrated that SS induces calcium entry into RBC, which might be required for certain responses linked with SS (e.g., nitric oxide generation) [43]. Mechano-sensitive calcium channels have been shown to exist in the RBC membrane [10] and to respond to local deformations of the membrane to increase calcium permeability [15]. Intracellular calcium concentration has long been considered an important determinant of RBC deformability $[5,18$, 32]. Further, extracellular calcium is a determinant of RBC sub-hemolytic damage under extremely high SS [35], although this finding could not be confirmed in experiments utilizing lower SS [9]. Intracellular calcium concentration may influence a variety of protein functions mediated by the calcium-calmodulin complex, including membrane skeletal proteins [41]. The calcium-dependent potassium channel, often termed the Gardos channel, might be another mediator of impaired deformability, leading to RBC dehydration with increased intracellular calcium concentration [25]. Calcium may also influence protein kinase $\mathrm{C}$ activity to mediate altered deformability [34]. It has been suggested that there is a reciprocal relation between calcium-calmodulin and cyclic adenosine monophosphate (cAMP)-related control mechanisms [31,35]. Therefore, it seems reasonable that $G$ protein-coupled receptor activation, cAMP levels and protein kinase A activity as related to the regulation of spectrin network-integral protein relationship is the dominant pathway $[30,35]$. The enhanced response in terms of deformability improvement under SS in the presence of the calcium-chelator EDTA might be explained by this reciprocal relationship. Further studies with manipulators of these intracellular signaling mechanisms are needed to test these hypotheses.

The extent of the increase in RBC deformability by shear stress was on the order of 5-7\% for various conditions (Table 1), and such an improvement of EI may have a significant influence on microcirculatory blood flow. It has been shown that an experimental decrease of RBC deformability corresponding to a $7.8 \%$ decrease in EI resulted in $\sim 80 \%$ increase of flow resistance in an isolated-perfused rat hindlimb preparation in which vasomotor reserve was abolished [1]. In the present study, the magnitude of the applied SS was found to be a determinant of the deformability response: at higher SS, the time constant 
was shorter and the change in EI was greater (Fig. 4). Shear stress levels in the circulatory system are a function of the pressure gradient in a given vascular segment. In turn, the pressure gradient may reflect flow resistance in that segment [36]. Therefore, the improvement in deformability under local SS at the magnitude observed in the current study may have significant effects on blood flow dynamics, at least in tissues supplied by blood vessels with impaired vasomotor reserve, and may therefore serve as a compensating mechanism for the maintenance of adequate tissue perfusion [4].

The observed improvement of RBC deformability in the current study is expected to reflect the response during each passage of RBC through the microcirculation; the improvement then fades when the RBC enter the venous circulation with lower SS. Although the influence of chronic exposure of RBC to higher levels SS cannot be estimated based on our observations, there are interesting clinical reports indicating improved RBC deformability in patients with increased arterial stiffness and hence higher SS in the vasculature [23]. This aspect of the possible influence of SS on RBC deformability should be evaluated by separate experimental studies.

It should also be kept in mind that SS influences the functions of other cells in the vasculature. The maintenance of the quiescent status of endothelial cells and their physiological functions (e.g., contribution to vasomotor control) depends on the continued exposure to SS at physiological levels and geometry [14]. It has been demonstrated that increased levels of SS in the vasculature within physiological range improve tissue perfusion by increasing NO generation by endothelial cells [44]. However, SS may also affect platelets in the circulation inducing their activation and aggregation, thereby increasing the tendency for thrombosis [37]. Therefore, the RBC response to SS seems to be an additional factor to consider with respect to the role of SS in the maintenance of adequate tissue perfusion. These considerations thus place RBC into a more active role in the functioning of the circulation, in contrast with their usually assumed classical passive role as an oxygen transporter. Such a change in physiological perspectives related to the role of the RBC and its function may yield new clinical and therapeutic approaches to circulatory disorders.

\section{Acknowledgements}

This study was supported by in part by NIH research Grants HL015722 and HL090511 and the Turkish Academy of Sciences.

\section{References}

[1] O.K. Baskurt, In vivo correlates of altered blood rheology, Biorheology 45 (2008), 629-638.

[2] O.K. Baskurt, M. Boynard, G.R. Cokelet, P. Connes, B.M. Cooke and S. Forconi, New guidelines for hemorheological laboratory techniques, Clin. Hemorheol. Microcirc. 42 (2009), 75-97.

[3] O.K. Baskurt, M.R. Hardeman, M. Uyuklu, P. Ulker, M. Cengiz, N. Nemeth et al., Parametrization of red blood cell elongation index-shear stress curves obtained by ektacytometry, Scand. J. Clin. Lab. Invest. 69 (2009), 777-788.

[4] O.K. Baskurt, E. Levi, S. Caglayan, N. Dikmenoglu, O. Ucer, R. Guner and S. Yorukan, The role of hemorheological factors in the coronary circulation, Clin. Hemorheol. 11 (1991), 121-127.

[5] O.K. Baskurt, E. Levi, A. Temizer, D. Ozer, S. Caglayan, N. Dikmenoglu and S.O. Andac, In vitro effects of thyroxine on the mechanical properties of erythrocytes, Life Sci. 46 (1990), 1471-1477.

[6] O.K. Baskurt and H.J. Meiselman, Data reduction methods for ektacytometry in clinical hemorheology, Clin. Hemorheol. Microcirc. 54 (2013), 99-107.

[7] O.K. Baskurt and H.J. Meiselman, Blood rheology and hemodynamics, Semin. Thromb. Hemost. 29 (2003), 435-450.

[8] O.K. Baskurt and H.J. Meiselman, Determination of red blood cell shape recovery time constant in a Couette system by the analysis of light reflectance and ektacytometry, Biorheology 33 (1996), 489-503. 
[9] O.K. Baskurt, M. Uyuklu and H.J. Meiselman, Protection of erythrocytes from sub-hemolytic mechanical damage by nitric oxide mediated inhibition of potassium leakage, Biorheology 41 (2004), 79-89.

[10] M.C. Brain, C. Pihl, L. Robertson and C.B. Brown, Evidence for a mechanosensitive calcium influx into red cells, Blood Cells Mol. Dis. 32 (2004), 349-352.

[11] F.A. Carvalho, J.P. de Almeida, T. Freitas-Santos and C. Saldanha, Modulation of erythrocyte acetylcholinesterase activity and its association with G protein-band 3 interactions, J. Membr. Biol. 228 (2009), 89-97.

[12] J.A. Chasis and N. Mohandas, Erythrocyte membrane deformability and stability: two distinct membrane properties that are independently regulated by skeletal protein associations, J. Cell. Biol. 103 (1986), 343-350.

[13] S. Chien, Red cell deformability and its relevance to blood flow, Ann. Rev. Physiol. 49 (1987), 177-192.

[14] S. Chien, Molecular basis of rheological modulation of endothelial functions: Importance of stress direction, Biorheology 43 (2006), 95-116.

[15] A. Dyrda, U. Cytlak, A. Ciuraszkiewicz, A. Lipinska, A. Cueff, G. Bouyer et al., Local membrane deformations activate $\mathrm{Ca}^{2+}$-dependent $\mathrm{K}^{+}$and anionic currents in intact human red blood cells, PLoS One 5 (2010).

[16] M.L. Ellsworth, C.G. Ellis, D. Goldman, A.H. Stephenson, H.H. Dietrich and R.S. Sprague, Erythrocytes: oxygen sensors and modulators of vascular tone, Physiology 24 (2009), 107-116.

[17] E. Ferru, K. Giger, A. Pantaleo, E. Campanella, J. Grey, K. Ritchie et al., Regulation of membrane-cytoskeletal interactions by tyrosine phosphorylation of erythrocyte band 3, Blood 117 (2011), 5998-6006.

[18] E. Friederichs, R.A. Farley and H.J. Meiselman, Influence of calcium permeabilization and membrane-attached hemoglobin on erythrocyte deformability, Am. J. Hematol. 41 (1992), 170-177.

[19] S.R. Goodman, A. Kurdia, L. Ammann, D. Kakhniashvili and O. Daescu, The human red blood cell proteome and interactome, Exp. Biol. Med. 232 (2007), 1391-1408.

[20] J.M. Greve, A.S. Les, B.T. Tang, M.T. Draney Blomme, N.M. Wilson et al., Allometric scaling of wall shear stress from mice to humans: quantification using cine phase-contrast MRI and computational fluid dynamics, Am. J. Physiol. Heart Circ. Physiol. 291 (2006), H1700-H1708.

[21] M.R. Hardeman, P.T. Goedhart and S. Shin, Methods in hemorheology, in: Handbook Hemorheology and Hemodynamics, O.K. Baskurt, M.R. Hardeman, M.W. Rampling and H.J. Meiselman, eds, IOS Press, Amsterdam, 2007, pp. $242-266$.

[22] M.V. Kameneva and J.F. Antaki, Mechanical trauma to blood, in: Handbook of Hemorheology and Hemodynamics, O.K. Baskurt, M.R. Hardeman, M.W. Rampling and H.J. Meiselman, eds, IOS Press, Amsterdam, 2007, pp. $206-227$.

[23] S.S. Lee, N.J. Kim, K. Sun, J.G. Dobbe, M.R. Hardeman, J.F. Antaki et al., Association between arterial stiffness and the deformability of red blood cells (RBCs), Clin. Hemorheol. Microcirc. 34 (2006), 475-481.

[24] L.B. Leverett, J.D. Hellums, C.P. Alfrey and E.C. Lynch, Red blood cell damage by shear stress, Biophys. J. 12 (1972), 257-273.

[25] Q. Li, V. Jungmann, A. Kiyatkin and P.S. Low, Prostaglandin E2 stimulates a $\mathrm{Ca}^{2+}{ }_{\text {-dependent }}{ }^{+}$channel in human erythrocytes and alters cell volume and filterability, J. Biol. Chem. 271 (1996), 18651-18656.

[26] H.H. Lipowsky, L.E. Cram, W. Justice and M.J. Eppihimer, Effect of erythrocyte deformability on in vivo red cell transit time and hematocrit and their correlation with in vitro filterability, Microvasc. Res. 46 (1993), 43-64.

[27] G. Minetti and P.S. Low, Erythrocyte signal transduction pathways and their possible functions, Curr. Opin. Hematol. 4 (1997), 116-121.

[28] N. Mohandas, M.B. Clark, M.S. Jacobs and S.B. Shohet, Analysis of factors regulating erythrocyte deformability, J. Clin. Invest. 66 (1980), 563-573.

[29] N. Mohandas, M.R. Clark, B.P. Health, M. Rossi, L.C. Wolfe, S.E. Lux and S.B. Shohet, A technique to detect reduced mechanical stability of red cell membranes: relevance to elliptocytic disorders, Blood (1982), 768-774.

[30] A.V. Muravyov and I.A. Tikhomirova, Role of molecular signaling pathways in changes of red blood cell deformability, Clin. Hemorheol. Microcirc. 53 (2013), 45-59.

[31] A.V. Muravyov, I.A. Tikhomirova, A.A. Mimistova, S.V. Bulaeva, A.V. Zamishlayev and E.A. Batalova, Crosstalk between adenylyl cyclase signaling pathway and $\mathrm{Ca}^{2+}$ regulatory mechanism under red blood cell microrheological changes, Clin. Hemorheol. Microcirc. 45 (2010), 337-345.

[32] E.A. O'Rear, M.M. Udden, L.V. McIntire and E.C. Lynch, Reduced erythrocyte deformability associated with calcium accumulation, Biochim. Biophys. Acta (BBA) - Biomembranes 691 (1982), 274-280.

[33] J.J. Olearczyk, A.H. Stephenson, A.J. Lonigro and R.S. Sprague, Heterotrimeric G protein Gi is involved in a signal transduction pathway for ATP release from erythrcytes, Am. J. Physiol. Heart Circ. Physiol. 286 (2004), H940-H945.

[34] S. Oliveira, A.S. Silva-Herdade and C. Saldanha, Modulation of erythrocyte deformability by PKC activity, Clin. Hemorheol. Microcirc. 39 (2008), 363-373.

[35] T. Oonishi, K. Sakashita and N. Uyesaka, Regulation of red blood cell filterability by $\mathrm{Ca}^{2+}$ influx and cAMP-mediated signaling pathways, Am. J. Physiol. Cell Physiol. 273 (1997), C1828-C1834.

[36] R. Reneman and A. Hoeks, Wall shear stress as measured in vivo: consequences for the design of the arterial system, Med. Biol. Eng. Comput. 46 (2008), 499-507. 
[37] D.A. Rubenstein and W. Yin, Quantifying the effects of shear stress and shear exposure duration regulation on flow induced platelet activation and aggregation, J. Thromb. Thrombolysis 30 (2010), 36-45.

[38] C. Saldanha, A.S. Silva, S. Goncalves and J. Martins-Silva, Modulation of erythrocyte hemorheological properties by band 3 phosphorylation and dephosphorylation, Clin. Hemorheol. Microcirc. 36 (2007), 183-194.

[39] H. Schlichting and K. Gersten, Boundary Layer Theory, Springer-Verlag, Berlin, 2000.

[40] R.S. Sprague, M.L. Ellsworth, A.H. Stephenson, M.E. Kleinhenz and A.J. Lonigro, Deformation-induced ATP release from red blood cells requires CFTR activity, Am. J. Physiol. Heart Circ. Physiol. 275 (1998), H1726-H1732.

[41] Y. Takakuwa and N. Mohandas, Modulation of erythrocyte membrane material properties by $\mathrm{Ca}^{2+}$ and calmodulin. Implications for their role in regulation of skeletal protein interactions, J. Clin. Invest. 82 (1988), 394-400.

[42] P. Ulker, H.J. Meiselman and O.K. Baskurt, Nitric oxide generation in red blood cells induced by mechanical stress, Clin. Hemorheol. Microcirc. 45 (2010), 169-175.

[43] P. Ulker, N. Yaras, O. Yalcin, C. Celik-Ozenci, P.C. Johnson, H.J. Meiselman and O.K. Baskurt, Shear stress activation of nitric oxide synthase and increased nitric oxide levels in human red blood cells, Nitric Oxide: Biology and Chemistry 24 (2011), 184-191.

[44] R. Varin, P. Mulder, V. Richard, F. Tamion, C. Devaux, J. Henry et al., Exercise improves flow-mediated vasodilation of skeletal muscle arteries in rats with chronic heart failure: role of nitric oxide, prostanoids and oxidant stress, Circulation 99 (1999), 2951-2957. 